\title{
La RDA dans le (rétro)viseur. Plaidoyer pour une autre perception
}

\section{Carola Hähnel-Mesnard}

\author{
Université de Lille
}

\section{Abstract}

Der Beitrag hinterfragt die Konstruktion und Vermittlung der Erinnerung an die DDR. Heutzutage ist die Interpretation der DDR-Geschichte in den Medien, in den meisten staatlichen Museen sowie in erfolgreichen Kinoproduktionen immer noch stark durch das totalitaristische Paradigma geprägt. Diese Darstellung steht im Widerspruch zur Alltagswahrnehmung der meisten ehemaligen DDR-Bürger. Obwohl sich die Forschung seit langem für Aspekte der Alltagsgeschichte interessiert, sind solche Elemente in den meisten aktuellen Darstellungen der DDR kaum vorhanden. Andreas Dresens und Laila Stielers Film Gundermann (2018) ist ein Beispiel für den Versuch von Ostdeutschen, die Deutungshoheit über ihre Geschichte zurückzugewinnen. Insgesamt beeinflusst die Unterrepräsentation der Ostdeutschen in Schlüsselpositionen der Gesellschaft auch die Möglichkeiten, auf die Darstellung dieser Vergangenheit einzuwirken.

Cette contribution s'interroge sur la façon dont la mémoire de la RDA est construite et transmise. Aujourd'hui, l'interprétation de l'histoire de la RDA se fait toujours essentiellement à partir du paradigme totalitaire, que ce soit dans les médias, dans la plupart des musées financés par l'État ou dans les productions cinématographiques à succès. Cette représentation est en décalage par rapport à la perception de la RDA telle qu'elle fut vécue de l'intérieur par la grande majorité de ses citoyens. Alors que la réflexion sur l'histoire du quotidien est présente depuis longtemps dans le monde de la recherche, les aspects qui en relèvent sont peu présents dans les représentations actuelles de la RDA. Le film Gundermann (2018) d'Andreas Dresen et Laila Stieler est un récent exemple d'une tentative entreprise par les Allemands de l'Est pour récupérer un pouvoir d'interprétation de leur passé. Globalement, la sous-représentation de ces derniers dans les positions-clés de la société influe également sur les possibilités de prise de parole sur ce passé.

This paper examines the construction and mediation of the GDR memory. Today, the interpretation of the GDR history in the media, through most of the state museums as well as successful movies, is still strongly influenced by the totalitarian paradigm. This representation contradicts the everyday perception of most former GDR citizens. Although research inspired by Alltagsgeschichte has long been interested in aspects of everyday history, such elements are scarcely present in most current representations of the GDR. Andreas Dresen's and Laila Stieler's film Gundermann (2018) is an example of East German attempts to regain sovereignty over the interpretation of their history. Overall, the under-representation of the East Germans in key positions in society also influences the possibilities of impacting the portrayal of this past.

\section{Keywords}

Darstellung der DDR • Totalitarismus • Alltagsgeschichte $•$ Gundermann • Deutungshoheit

représentation de la $\mathrm{RDA} \cdot$ totalitarisme $\bullet$ histoire du quotidien $•$ Gundermann • pouvoir d'interprétation

Representation of GDR • totalitarism • Alltagsgeschichte $•$ Gundermann • interpretative authority

Lorsque l'on se promène, en 2019, dans le centre-ville de Sofia et qu'on parcourt le très beau boulevard Vitocha, on tombe sur un grand parc au fond duquel se dresse une gigantesque bâtisse de l'époque socialiste : c'est l'ancien Palais de la Culture construit à la fin des années 1970 et qui sert aujourd'hui de centre de congrès et de salle de spectacles. Quand la Bulgarie assurait la présidence du Conseil de l'Union Européenne en 2018 , les rencontres officielles avaient lieu dans ce bâtiment. Lorsque l'on se promène, en 2019, dans le centre-ville de Berlin et qu'on parcourt le boulevard Unter den Linden, pas moins beau que le précédent, on tombe sur un gigantesque bâtiment d'une époque incertaine se donnant

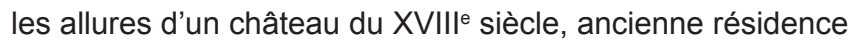
de la dynastie des Hohenzollern, symbole de tout ce que la monarchie absolue de Prusse avait de « meilleur ». Gare au visiteur qui, n'étant pas venu à Berlin depuis des années, se souvient encore y avoir vu, au même endroit, une autre gigantesque bâtisse, de l'époque socialiste, construite à la fin des années 1970. Incrédule, confrontant ses photos de l'époque à la nouvelle configuration, il cherchera en vain la moindre plaque lui indiquant qu'entre 1976 et 2006 se dressait très officiellement, ici même, le Palast der Republik, et il 
conclura qu'il aura été victime d'hallucinations. Ou bien que les appareils photos de l'époque n'étaient vraiment pas fiables.

Retournement de l'histoire ? Effacement des traces ? L'occasion d'un regard comparatif a soudainement fait ressurgir cet effacement de l'histoire et du patrimoine est-allemands qui a déjà fait l'objet de nombreux commentaires (Robin 2001 ; Combe / Dufrêne / Robin 2009 ; Goudin 2017) Je n'y reviendrai pas. Au moins, me dis-je, les Sofiotes n'ont pas été dessaisis de cette partie de leur histoire, et le jour où la grande bâtisse de l'époque socialiste ne leur conviendra plus, ils décideront de son sort par eux-mêmes.

Dessaisis de leur histoire - d'aucuns diront qu'il s'agit là de bien grands mots. Et pourtant, si on cherche aujourd'hui les raisons du malaise perceptible dans les nouveaux Länder s'exprimant dans un vote massif pour le parti d'extrême droite et populiste AfD, et si on se désole que l'« unité intérieure » soit loin d'être acquise trente ans après la chute du Mur, on doit également s'interroger sur la façon dont les traces de la RDA persistent ou non dans l'espace public, sur la façon dont sa mémoire est construite et sur la possibilité qu'ont d'ex-citoyens est-allemands d'y participer, et on doit aussi s'interroger tout court sur la façon dont l'Est peut s'impliquer dans la société allemande toute entière.

\section{DU SPECTRE DU TOTALITARISME AU SENTIMENT D'INADÉQUATION DE LA MÉMOIRE}

En septembre 2018, le journal d'Arte a consacré un court reportage à la sortie de la BD Spirou in Berlin du dessinateur allemand Flix (Frank 2018). Avant d'envoyer ses héros Spirou et Fantasio vivre quelques aventures dans le BerlinEst des années 1980, on nous apprend que Flix prend la peine de se documenter. Pour pouvoir utiliser un motif récurrent de la $B D$, les singes dressés, le dessinateur découvre que " les singes dressés ont aussi joué un rôle en RDA »! En effet, " il y avait un élevage de singes dans le Tierpark à Berlin ». Jusque-là, rien d'extraordinaire pour un zoo, aussi communiste soit-il. Évidemment, la réflexion va au-delà : «En même temps, le singe dressé, c'est un peu le symbole du peuple dans un système politique fermé comme l'était la RDA. » Soit ! Ensuite, Flix nous confie avoir " essayé de rechercher précisément les menaces qui pesaient sur les citoyens de la RDA. Une des choses qui pouvait leur arriver s'ils ne se comportaient pas de façon conforme aux attentes, c'était d'être emprisonné. Dans les prisons, ils étaient soumis à une torture psychique et physique. " Pendant ce temps-là, Flix est filmé dans la prison de la Stasi à Hohenschönhausen, la voix-off met encore l'accent sur le mot « torture ». On aurait aimé avoir un peu plus d'informations sur les circonstances précises. Et qu'est-ce que cela veut dire : " ne pas se comporter de façon conforme aux attentes » pour aller en prison ? Le ton est donné, de même que la trame d'un récit qui n'a de cesse de se répéter depuis trente ans : les Allemands de l'Est, qui ont l'échine souple comme les singes, sont les victimes d'un système totalitaire qui les emprisonne à la moindre occasion. Pour Flix, né en 1976 à Münster en République fédérale, sa courte confrontation avec l'histoire de la RDA s'arrête là.

Pourquoi d'ailleurs s'attarder sur ce petit reportage d'à peine deux minutes passé sur la chaîne culturelle franco-allemande ? Parce qu'il est parfaitement représentatif d'un discours condescendant ainsi que d'une vision biaisée et manichéenne de l'histoire est-allemande. Parce qu'il fait preuve d'un relativisme historique de plus en plus courant. Recourir de façon imprécise au paradigme totalitaire dans la moindre des circonstances revient à nier les différences entre la terreur d'un régime totalitaire, en occurrence national-socialiste ou stalinien, et le système de contrôle et de surveillance dans une dictature autoritaire, avec comme résultat de relativiser le premier.

Dans son dernier livre, un virulent et stimulant règlement de compte avec l'Allemagne unifiée, l'essayiste est-allemande Daniela Dahn dénonce l'utilisation indifférenciée et récurrente du terme " totalitaire » appliqué à la RDA (Dahn 2019 : 101) et rappelle utilement les propos de Hannah Arendt dans sa préface à la troisième édition du Système totalitaire en 1966, à savoir qu'il faut " distinguer les différents types de dictatures communistes à parti unique [...] du véritable régime totalitaire ", de même que nous avons " toutes les raisons d'utiliser le mot ,totalitaire' avec parcimonie et prudence. " (Arendt 1972 : 12, 13). De son côté, I'historienne Sonia Combe déplore une mémorialisation du passé de la RDA qui a de plus en plus tendance à mettre sur un pied d'égalité la terreur nazie et la dictature est-allemande et à suggérer des continuités de 1933 à 1989, comme c'est le cas du mémorial de la prison de la Stasi à Hohenschönhausen évoqué plus haut (Combe 2019).

La mémoire de la RDA et sa représentation dans les musées et mémoriaux font depuis longtemps l'objet de débats. Disons qu'il s'agit toujours du même débat qui ne conduit à aucun changement d'optique. En effet, la plupart des musées et lieux de commémoration sont les anciens lieux du pouvoir, de la surveillance et de la répression : musées de la Stasi, prisons, Mur de Berlin et postes frontières. C'est le constat fait en 2006 par l'historien Martin Sabrow, chargé à l'époque d'élaborer un projet global pour conceptualiser le traitement du passé de la dictature du SED (Sabrow et al. 2007 : 17-43). Selon lui, il faut aller au-delà d'une telle approche, donner plus de place à la vie quotidienne et interroger les comportements individuels des acteurs de la société dans leurs relations avec le pouvoir, des comportements qui pouvaient osciller entre « acceptation et révolte, enthousiasme et mépris, résignation loyale et bonheur trouvé dans une ,niche“" (ibid. : 31sq.). En cela, Sabrow se fait le porte-parole d'une recherche universitaire qui, dès les années 1990, tendit à dépasser la seule histoire politique fortement dominée par les théories sur le totalitarisme et s'intéressant exclusivement aux institutions et aux structures du pouvoir : il s'agissait à l'inverse de privilégier l'histoire sociale et l'histoire du quotidien qui renoncent à l'idée d'une société monolithique et présupposent l'existence d'un écart entre la volonté de domination et l'expérience vécue 
(Lindenberger 1999 ; Kott / Droit 2006). Ainsi, Sabrow propose de s'intéresser aux forces d'adhésion (Bindungskräfte) grâce auxquelles le régime a pu acquérir une certaine stabilité dans les années 1960 et 1970, que ce soit par la conviction politique, d'un côté, ou par les possibilités d'ascension sociale et de sécurité économique de l'autre (Sabrow et al. 2007 : 34). Ces approches ne remettaient aucunement en question la mémoire des victimes de la répression du régime, elles ajoutaient simplement un pilier permettant d'exprimer les contradictions inhérentes au système politique et de proposer un espace de réflexion à ceux - probablement la grande majorité de la population - qui considéraient avoir mené une « vie normale » en RDA (Fulbrook 2008), tout en empêchant une idéalisation du passé a posteriori, ce qu'on a appelé « ostalgie ».

Les recommandations de Sabrow n'ont pas été suivies, on a reproché à la commission qu'il présidait de minimiser la gravité des actes commis par le régime est-allemand. La Stasi caractériserait mieux la RDA que les crèches, tel fut le verdict prononcé par Horst Möller, à l'époque directeur de l'Institut du temps présent de Munich (ibid. : 56). Depuis, le débat n'a pas avancé. Lorsqu'en 2013, le politologue Klaus Christoph fait le tour de la question, il constate que les rapports du gouvernement sur le sujet se ressemblent d'un débat parlementaire à l'autre, que la politique de la mémoire met toujours l'accent sur le caractère inhumain de la dictature communiste et sur le dualisme entre victimes et bourreaux. Ce politologue souligne, dans le sillage de Sabrow, que la majorité de la population est-allemande ne se reconnaît pas dans ce schéma et que les expériences individuelles sont le plus souvent en décalage avec la vision de l'histoire véhiculée par les instances politiques. Et de pointer une des conséquences de cette absence de nuances aujourd'hui : il est possible que cela rappelle aux Allemands de l'Est une expérience fondamentale faite en RDA, à savoir la mise en scène par le SED d'une réalité qui n'avait que peu à voir avec leur propre vécu (Christoph 2013).

En 2019, ce sentiment de décalage s'exprime encore autrement, dans une forte adhésion à des discours anti-élites et antidémocratiques où il n'est pas rare que l'Allemagne actuelle soit comparée à la RDA. Dans un récent livre dans lequel le sociologue Wolfgang Engler et la journaliste Jana Hensel essayent d'expliquer l'expérience est-allemande des trois dernières décennies, Hensel évoque la marginalisation des Allemands de l'Est quand il s'agit d'interpréter leur histoire, ce sentiment que « le récit de leur propre histoire est effectué par d'autres » (Engler / Hensel 2018 : 192). Actuellement, on observe un petit changement de perspective dans la mesure où la recrudescence du vote d'extrême droite dans les nouveaux Länder oblige à s'interroger sur les raisons qui y ont conduit. Et puisque trente ans nous séparent désormais d'une RDA qui n'en a vécu que quarante, on commence à admettre que les raisons de ce vote ne sont plus à chercher dans l'histoire de la RDA, mais dans les expériences faites depuis 1990 (ibid. : 51). Reste à savoir si les responsables politiques vont également admettre des erreurs quant aux usages de la mémoire de la RDA et tirer les conséquences de ces erreurs au plan mémoriel.

\section{RÉCITS HÉROÏQUES VERSUS MÉMOIRE DU QUOTIDIEN}

Un important vecteur de la mémoire de la RDA sont la télévision et le cinéma (Camarade / Guilhamon et al. 2018). Là encore, nous l'avons vu, la représentation de la RDA est rarement nuancée et peut susciter auprès du public est-allemand un sentiment d'étrangeté. Dans un entretien de 2016, le cinéaste est-allemand Thomas Heise affirme que la Stasi a trop longtemps dominé l'image de la RDA, et que la RDA est toujours perçue comme un « bloc monolithique sans histoire propre ", alors que la réalité n'était pas la même en 1949 ou en 1989 (Steinle 2018 : 304).

Parmi les films qui rencontrent de grands succès commerciaux, y compris à l'étranger, on trouve en effet des longsmétrages sur la Stasi comme La Vie des autres (2006), qui date déjà, mais également des récits héroïques de résistance au régime ou de tentatives d'évasion pour fuir la RDA. Sont ainsi récemment sortis La révolution silencieuse (Das schweigende Klassenzimmer) de Lars Kraume (2018) et Le vent de la liberté (Ballon) de Michael Herbig (2018). Le premier raconte l'organisation d'une minute de silence dans une classe de terminale, protestant ainsi contre la répression soviétique du soulèvement de 1956 en Hongrie. Si la représentation du rapport entre citoyens et pouvoir n'est pas dénuée de nuances, le film joue en revanche d'emblée, et délibérément, à travers des clichés, sur des analogies entre le pouvoir est-allemand et les nationaux-socialistes - encore un rapprochement tout à fait problématique des deux régimes. Quant au deuxième film, il raconte l'évasion en montgolfière de deux familles est-allemandes, une histoire qui a déjà fait l'objet d'une adaptation cinématographique par Disney au début des années 1980. Comme son collègue Lars Kraume, l'acteur et humoriste Michael Herbig est né en République fédérale. Leurs films respectifs reposent sur des " histoires vraies », ce qui donne ainsi aux récits la garantie d'une certaine authenticité. Mais ces films sont loin de pouvoir représenter le quotidien d'un citoyen de RDA, ils versent dans le sensationnel et l'extraordinaire, et l'Allemand de l'Est lambda aura le plus grand mal à s'y reconnaître. La grande majorité d'entre eux évitait de se frotter de trop près au pouvoir ou de mettre en danger sa vie lors de tentatives d'évasion. Certes, on est au cinéma et c'est le propre de ce média de raconter des histoires extraordinaires, de faire rêver et de provoquer des frissons. Sauf que, l'esthétique réaliste et rétro aidant, ce type de film est le plus souvent perçu comme un document historique de l'époque, comme la représentation adéquate et exhaustive d'une période du passé. Les protagonistes, aussi beaux que le décor soigneusement reconstruit, suscitent l'empathie et permettent au spectateur de s'identifier à eux (Mesnard 2018 : 10). En Allemagne et en France, ces films, souvent banals d'un point de vue cinématographique et esthétique, sont même utilisés à des fins pédagogiques, accompagnés de livrets didactiques, et 
transmettent une certaine image du passé à des générations d'élèves. Si les supports changent en fonction des dernières productions qui sortent sur le marché, la trame narrative et le ton restent toujours les mêmes : la RDA était un État répressif que ses habitants cherchaient à fuir. Dès les années 1960, l'imaginaire occidental relatif à la RDA s'était construit autour des mots-clés " mur " et « fuite ", et la majorité des films récents en sont encore le miroir. D'autres questions sur la période n'émergent même pas.

Ainsi, on ne s'intéresse pas à la façon dont les gens ont vécu au quotidien, quelles étaient leurs préoccupations dans cette société fermée, leurs rapports au pouvoir en dehors d'une confrontation directe, comment ils se sont arrangés avec cette situation de liberté limitée ou de contraintes relatives, et où ils ont pu trouver leurs propres marges de liberté. On retrouve les mêmes problématiques évoquées plus haut à propos de la mémorialisation de la RDA. La RDA n'était pas ce pays en noir et blanc, ou tout en gris, comme il est présenté en général. Ce qui manque à ce cinéma à succès prétendant retracer le passé de la RDA, c'est la représentation d'une mémoire du quotidien et des rapports sociaux. Le cinéma est-allemand lui-même est à ce titre une formidable source. Qu'il s'agisse de films de fiction ou de documentaires, les productions de la DEFA représentent « une mine essentielle pour qui souhaite réfléchir non pas seulement sur la mémoire de la RDA mais aussi sur la RDA telle qu'elle fut ,vécue de l'intérieur' ", comme Caroline Moine l'a fait remarquer il y a quelque temps déjà (Moine 2006 : 171).

Les films récents représentant la RDA « vécue de l'intérieur » sont rares, comme ceux qui s'intéressent aux conséquences sociales de la réunification. Et ils sont bien moins visibles que les autres. Alors que La Vie des autres, premier long métrage de Florian Henckel von Donnersmarck, attirait des millions de spectateurs en 2006, la comédie sociale Du bist nicht allein de Bernd Böhlich, sortie en 2007, a certes eu un petit succès en Allemagne, mais n'a pas été reçue ailleurs. Évidemment, les nouveaux chômeurs dans les grands ensembles de Berlin-Est fascinent moins que le pathétique officier de la Stasi. C'est encore vrai de deux films importants qui sont sortis en 2018, donc au même moment que les récits héroïques évoqués plus haut. On remarquera d'ailleurs l'origine, très parlante, des réalisateurs en fonction du sujet choisi : l'Est pour les films à caractère social, l'Ouest pour les autres. Le long-métrage In den Gängen (Une valse dans les allées) de Thomas Stuber, basé sur un récit de Clemens Meyer, est filmé presque exclusivement dans le huis clos d'un hypermarché dans la province est-allemande où le protagoniste Christian s'initie à son métier de cariste. La taille du magasin et les longues allées remplies de marchandises sont l'inverse symétrique de l'étroitesse d'un quotidien et d'une activité professionnelle perdant tout sens, si l'on excepte les relations humaines qui s'y nouent. Ainsi, Christian fait la connaissance de Bruno, un employé bien plus âgé qui le prend sous son aile. C'est ce personnage à première vue extrêmement stable qui résume à lui seul toute la violence des changements intervenus après 1989. Autrefois routier en RDA, il a perdu son emploi et fait savoir à Christian à quel point la route lui manque. Rien de plus n'est dit, mais son sentiment de liberté sur la route est discrètement opposé à la nouvelle liberté de consommer. Bruno, chef de rayon, apparemment bien intégré, va se suicider, alors que personne ne s'y attend. Outre le fait de brosser un tableau des classes inférieures dans la province est-allemande d'aujourd'hui, le film ne suggère rien d'autre que la chose suivante : la vie en RDA pouvait faire sens et l'implosion de l'ancien cadre de vie laisse des traces, même longtemps après sa disparition.

Le véritable film-événement dans notre contexte est Gundermann (2018) du réalisateur Andreas Dresen et de la scénariste Laila Stieler. Non commercialisé en France, il est projeté uniquement à de rares occasions, comme par exemple actuellement dans le cadre des commémorations des trente ans de la chute du Mur. Film donc que probablement aucun enseignant français n'utilisera dans ses cours d'histoire ou d'allemand. Évidemment, l'histoire est complexe et ne se réduit pas aux schémas en noir et blanc auxquels le public a été habitué. Ce film biographique retrace la vie de l'auteurcompositeur-interprète est-allemand Gerhard Gundermann (1955-1998) qui, dans la " vraie vie », est conducteur d'une immense excavatrice dans une mine de lignite à ciel ouvert. Ce long métrage permet une vue de l'intérieur de la société est-allemande avant la chute du Mur, tout en se focalisant sur la période de transformation des années 1990.

Gundermann est présenté comme un personnage aussi polyvalent qu'ambivalent : simple ouvrier qui fait les trois-huit, artiste le reste du temps, récalcitrant face aux fonctionnaires du parti et adhérant pourtant à la cause du socialisme, exclu du parti et recruté par la Stasi à laquelle il présente ses cahiers de doléances sur les conditions de travail dans la mine jusqu'à ce que celle-ci finisse par renoncer à ses services et le surveiller lui-même. Lorsque sa collaboration avec la Stasi devient publique dans les années 1990, il refuse d'admettre avoir été victime ou bourreau. L'un des objectifs du film est précisément d'abandonner les grilles de lecture simplistes, de refuser de porter un jugement définitif et de rendre aux biographies est-allemandes leur complexité. Gundermann est un film important, car une scénariste et un réalisateur est-allemands, nés dans les années 1960, se réapproprient l'histoire de la RDA pour la raconter à leur façon. Las d'être confrontés en permanence aux clichés et stéréotypes, ils ont souhaité se réemparer du monopole d'interprétation de leur propre histoire, comme Dresen l'explique dans le livret qui accompagne le DVD. Un film pour lequel il a d'ailleurs été très difficile de trouver des financements et un producteur. Non seulement le chansonnier Gundermann était absolument inconnu du public ouest-allemand, mais la dramaturgie particulière et le scénario, en refusant la repentance du personnage, allaient à l'encontre des conventions du genre (Dresen / Stieler 2018). Inutile de rappeler que Le vent de la liberté n'avait pas les mêmes problèmes.

Gundermann n'est pas seulement un film sur la façon qu'a un individu de se positionner face à l'idée socialiste ainsi que sur la manière dont les représentants du pouvoir la pervertissent, c'est également un film qui retrace minutieusement le monde du travail en RDA, les difficultés rencontrées pour 
respecter les objectifs du plan économique avec du matériel parfois vétuste, le travail en équipe, la satisfaction de réussir à chauffer le pays malgré une matière première de mauvaise qualité... Particulièrement touchant, le personnage de Helga, une petite femme toute frêle qui guide majestueusement l'immense excavatrice, renvoie au rôle des femmes dans ce milieu ouvrier. Quant à Gundermann, il ressent le besoin d'aller travailler dans sa mine avant et après ses concerts. Même quand il devient plus connu au début des années 1990 et aurait pu vivre de sa musique, il veut garder sa liberté face à l'industrie du spectacle. Lui et ses collègues seront particulièrement atteints par la fermeture de la mine et par le chômage qui s'ensuit. Ainsi, le film passe de la représentation du monde du travail en RDA à sa dissolution dans les années 1990, l'industrie des nouveaux Länder ne survivant que très rarement à l'arrivée de la nouvelle économie de marché. Et à Gundermann d'exprimer, dans une de ses chansons qui rythment le film, ce sentiment de déclassement et de désespoir qui caractérise l'après-1989 et qui, aujourd'hui, s'est transformé en ressentiment : "C'est ici que je suis né, là où les vaches sont aussi maigres que le bonheur / [...] / c'est ici que les nuls restent entre eux / ici, ce n'est pas mieux aujourd'hui qu'hier / et ici il n'y a pas de lendemain ». La chanson originale, C'est ici que je suis né (Hier bin ich geboren) date de 19951. Peut-être le cinéma aurait-il dû s'intéresser plus tôt à ces antihéros, tel Gundermann qui, avec ses immenses lunettes et sa queue de cheval dépassée, n'invite pas à une identification facile, mais qui incarne mieux que nos autres héros le cadre de vie d'une époque, avec ses contraintes, ses marges de manœuvre et les choix individuels qui y ont été effectués. Et aussi un antihéros qui incarne la disparition de ce cadre de vie, ainsi que la désolation et le mutisme qui en furent la conséquence.

\section{RETROUVER SA VOIX}

Dans le générique de Material de Thomas Heise, un documentaire de 2009 monté à partir des rushes de ses films précédents, le cinéaste affirme : " II y a toujours quelque chose qui reste, un reste qui ne se résout pas, alors les images sont là, éparses, et attendent d'être mises en histoire ${ }^{2}$. II y a toujours des images qui se soustraient à un récit, au grand récit de l'histoire, elles attendent d'être retrouvées pour raconter leurs histoires à elles, et l'histoire à leur tour. Peutêtre faut-il commencer à raconter l'histoire de façon quelque peu différente ? La phrase de Heise fait quelque part penser au chiffonnier de Walter Benjamin, celui qui s'intéresse au rebut de l'histoire, à ce qui en a été exclu. Et qui, en tant qu'historien, s'intéresse aux « opprimés » de l'histoire...

Ne filons pas la métaphore trop loin, les Allemands de l'Est ne sont pas les victimes de l'histoire. Aux premières élections

\footnotetext{
1 " Hier bin ich geborn / wo die Kühe mager sind wie das Glück /[...]/ hier sind die Nullen ganz unter sich/hier isses heute nicht besser als gestern/und ein Morgen gibt es hier nicht $[\ldots]$..

2 « Immer bleibt etwas übrig, ein Rest, der nicht aufgeht, dann liegen die Bilder herum und warten auf Geschichte.»
}

libres de mars 1990, la majorité d'entre eux, attirés par les promesses de transformation rapide, d'introduction du DMark et d'accès à la consommation, votent pour l'alliance des partis conservateurs et ouvrent ainsi la voie à un processus d'unification extrêmement rapide dont on paie aujourd'hui encore les conséquences. La majorité ne vote donc pas pour les représentants des mouvements de citoyens pour qui l'unification des deux Allemagnes n'était pas une option pertinente et qui cherchaient à réformer le socialisme, à trouver une troisième voie. Mais à l'époque, la majorité ne pouvait ou ne voulait certainement pas imaginer que la contrepartie du D-Mark résidait dans les transformations profondes qui allaient faire basculer leurs vies. Et cette majorité n'imaginait pas non plus que l'économie sociale de marché dont l'image leur était familière, à eux qui étaient de fidèles observateurs de la République fédérale, allait rapidement perdre l'épithète " social " sous les effets d'une mondialisation de plus en plus sauvage. Le rôle de la Treuhand interroge aujourd'hui, notamment le bradage de l'industrie est-allemande et leur corollaire : la désindustrialisation de ces régions, la montée du chômage, la dépopulation.

Ce que la majorité des Allemands de l'Est auraient pu voir à l'époque, c'est que, déjà, ils n'étaient plus tout à fait seuls à se prononcer sur leur avenir et à en déterminer les options. En effet, comme le décrit le sociologue Steffen Mau, lors de ces premières élections démocratiques en mars 1990, « sur les places publiques de RDA, les principaux orateurs venaient d'Allemagne de l'Ouest » - comme d'ailleurs aujourd'hui la plupart des cadres de l'AfD -, et les nouveaux partis indépendants n'avaient aucune chance en face, d'un côté, du SPD et de la CDU financés et soutenus par leurs camarades ouest-allemands qui dominaient largement la campagne électorale et, de l'autre côté, du PDS récemment créé sur les décombres du SED (Mau 2019 : 122). Est-ce que les Allemands de l'Est auraient voté autrement sans cette très forte et évidente ingérence ? Rien n'est moins sûr. Rétrospectivement, c'est néanmoins le moment où les représentants des mouvements de citoyens ayant déclenché le mouvement démocratique et le processus de réformes ont été dépossédés des utopies qui étaient les leurs et dont on se souvient à peine aujourd'hui. Et c'est le moment où les Allemands de l'Est ont commencé à perdre le contrôle des changements qui les concernaient pourtant en premier lieu.

L'un des problèmes évoqués pour expliquer le comportement électoral dans les nouveaux Länder et le ressentiment qui y fait rage est la question relative à la dévalorisation des expériences de vie et au remplacement des élites. Phénomène connu et vécu au quotidien, mais que l'on semble redécouvrir aujourd'hui. Daniela Dahn a récemment donné nombre d'exemples déconcertants : des expertises infondées de soi-disant spécialistes sonnant le glas d'institutions est-allemandes, ou appelant au remplacement de cadres, etc. (Dahn 2019 : 68-83). Le sociologue Raj Kollmorgen a fait le bilan de cette évolution désastreuse : il estime que les Allemands de l'Est ne représentent que $25 \%$ des élites sur leur propre territoire, dans les nouveaux Länder. Quant aux $75 \%$ restants : ils viennent de l'Ouest. Au niveau fédéral, la représentativité est d'environ $3 \%$. Plus la position est élevée - juge fédéral, 
général d'armée -, moins on trouve de représentants estallemands (Kollmorgen 2017 : 57sq.). Les Allemands de l'Est sont donc minoritaires dans les positions-clé de leur administration, de la justice, de l'armée, des médias, des entreprises et des universités. Kollmorgen souligne par ailleurs le phénomène d'auto-marginalisation : les jeunes Allemands nés à l'Est n'ont même plus l'ambition d'occuper des positions importantes (ibid. : 62).

Cette situation de non-représentativité dans les instances décisives - Angela Merkel restera, comme Joachim Gauck, l'exception à la règle - induit forcément une absence de parole proprement est-allemande qui se traduit aussi au niveau de la perception de l'Est dans l'espace public. Même les lieux culturels avec, à l'origine, une forte identité est-allemande véhiculent aujourd'hui le plus souvent une parole venant de Munich, de Hambourg ou de Londres. On se souvient des débats autour de la succession de Frank Castorf à l'intendance de la Volksbühne de Berlin, on a récemment évoqué le festival du film documentaire de Leipzig qui, depuis 2004, n'a plus eu de direction originaire de l'Est. Comme le rappelle le journaliste Matthias Dell, on pourrait arrêter de regarder en permanence les origines des uns et des autres si les institutions équivalentes ouest-allemandes étaient tout naturellement dirigées par des Allemands de l'Est, mais ce n'est pas le cas (Dell 2019).

II y a une violence symbolique de non-reconnaissance qui pèse sur l'Est de l'Allemagne. Et il y a un poids lié à l'indifférence et à l'ignorance. La plupart des Allemands de l'Ouest se sentent finalement peu concernés par ces régions de l'Est, de même que la chute du Mur ou la période de transformations ont peu influencé le cours de leur vie. L'histoire de la RDA comme celle de sa culture et de sa littérature sont aujourd'hui probablement plus enseignées dans les universités de France ou des États-Unis qu'en Allemagne même. Et on sait finalement peu de choses sur la révolution pacifique de 1989. Peut-être la crise politique actuelle déclenchée par la montée en puissance de l'extrême droite dans les nouveaux Länder conduira-t-elle à une prise de conscience. Des ouvrages récemment parus essaient d'expliciter, encore et encore, le regard est-allemand sur les trente dernières années qui nous séparent de 1989 (Engler / Hensel 2018 ; Dahn 2019 ; Mau 2019).

En 1990, l'essayiste Lothar Baier faisait remarquer que les Allemands de l'Ouest trouvaient sur le territoire de la RDA un espace dans lequel ils pouvaient rattraper (à peu de frais) une partie de l'histoire coloniale qu'ils n'avaient pas eue, sans faire l'effort de la conquête et sans payer de prix fort (Baier $1990: 105)^{3}$. En même temps, il mettait en garde contre l'effet « boomerang » qu'une " colonisation rapide » de la RDA pourrait provoquer à l'avenir (ibid. : 111). Effet « boomerang » que, semble-t-il, nous ressentons pleinement en ce moment. On pourrait recourir à la comparaison coloniale ou postcoloniale dans une autre perspective ; c'est une interrogation qui sous-tend ces lignes depuis le début. En effet, on pourrait se demander avec Gayatri Spivak : les Allemands de l'Est « peuvent-ils parler » ? Can the Subaltern speak ? Être assigné à la place du subalterne est un sentiment répandu à l'Est, paradoxalement même là où ce subalterne réussit. Kollmorgen rappelle qu'encore aujourd'hui, les Allemands de l'Est et l'Allemagne de l'Est sont considérés comme étant inférieurs à l'Ouest (Kollmorgen 2017 : 64). L'absence de visibilité positive dans l'espace public et l'impression que l'autre parle toujours à votre place, le sentiment d'être stigmatisé et regardé de façon condescendante, tout cela a des conséquences sur la perception de soi-même. Pour le moment, les « subalternes » parlent surtout à travers les urnes, il est temps qu'ils récupèrent une place visible dans la société pour utiliser leur voix autrement et de façon constructive, à condition évidemment qu'on les laisse parler. 3 Cette " thèse de la colonisation » a été très présente dans la littérature juste après la
chute du Mur, défendue par des auteurs est-allemands comme Volker Braun, Christoph chute du Mur, défendue par des auteurs est-allemands comme Volker Braun, Christoph
Hein, Stefan Heym, Heiner Müller et Christa Wolf, mais aussi par des auteurs ouest-alleHein, Stefan Heym, Heiner Müller et Christa Wolf, mais aussi par des auteurs ouest-alle-
mands comme Günter Grass, Rolf Hochhuth ou encore F.C. Delius (Born 2019: 94116 , 218).

\section{Bibliographie}

Arendt, Hannah (1972), Les origines du totalitarisme. Le système totalitaire, traduit de l'américain par Jean-Loup Bourget et al., Paris, Éditions du Seuil.

Baier, Lothar (1990), Volk ohne Zeit. Essay über das eilige Vaterland, Berlin, Wagenbach.

Camarade, Hélène / Guilhamon, Elizabeth / Steinle, Matthias / Yèche, Hélène (dir.) (2018), La RDA et la société postsocialiste dans le cinéma allemand après 1989, Villeneuve d'Ascq, Septentrion.

Born, Arne (2019), Literaturgeschichte der deutschen Einheit 19892000. Fremdheit zwischen Ost und West, Hannover, Wehrhahn Verlag.

Christoph, Klaus (2013), " ,Aufarbeitung der SED-Diktatur' - heute so wie gestern? - Essay », in Aus Politik und Zeitgeschichte, 4243/2013 (https://www.bpb.de/apuz/170166/aufarbeitung-der-seddiktatur-heute-so-wie-gestern?p=all\#fr-footnode32, consulté le 01/11/2019)
Combe, Sonia (2019), « Usages et mésusages de la mémoire en Allemagne », in Le Monde, 28/02/2019.

Combe, Sonia / Dufrêne, Thierry / Robin, Régine (2009), Berlin : l'effacement des traces, 1989-2009, Paris, BDIC/Fage Éditions.

Dahn, Daniela (2019), Der Schnee von gestern ist die Sintflut von heute. Die Einheit - eine Abrechnung, Hamburg, Rowohlt Taschenbuch Verlag.

Dell, Matthias (2019), « Ohnmachtspolitik. Ein Leipziger Lehrstück », in Cargo. Film, Medien, Kultur, 30/10/2019 (https://www.cargofilm.de/festival/dok-leipzig/dok-leipzig-2019/dok-leipzig-2019/, consulté le 04/11/2019).

Dresen, Andreas / Stieler, Laila (2018), « Es gibt keine Absolution, Punkt », interview avec Katja Nicodemus et Hanno Rauterberg, in Die Zeit, 03/10/2018 (https://www.zeit.de/2018/41/andreasdresen-laila-stieler-gundermann-interview /komplett ansicht, consulté le 03/11/2019). 
Engler, Wolfgang / Hensel, Jana (2018), Wer wir sind. Die Erfahrung, ostdeutsch zu sein, Berlin, Aufbau Verlag.

Fulbrook, Mary (2008), Ein ganz normales Leben. Alltag und Gesellschaft in der DDR, Darmstadt, WGB.

Frank, N. (2018), « Spirou à Berlin », 21/09/2018 (https://www.arte.tv/ fr/videos/085002-000-A/spirou-a-berlin/, consulté le 01/11/2019).

Goudin, Élisa (2017), « Destruction du Palais de la République et reconstruction du château des Hohenzollern : quelle place pour les considérations d'éthique publique dans la gestion du patrimoine berlinois ? ", in Éthique publique, Revue internationale d'éthique sociale et gouvernementale, (2) 19, (https://journals.openedition. org/ethiquepublique/2966, consulté le 30/10/2019).

Kollmorgen, Raj (2017), « Ostdeutsche in den Eliten. Problemdimensionen und Zukunftsperspektiven », in Ostdeutsche Eliten. Träume, WirklichkeitenundPerspektiven, hrsg.v. Deutsche Gesellschafte.V., Berlin, 54-65 (https://www.deutsche-gesellschaft-ev.de/images/veranstaltungen/konferenzen-tagungen/2017-pb-ostdeutsche-eliten/ Deutsche_Gesellschaft_eV_Broschuere_Ostdeutsche_Eliten.pdf, consulté le 03/11/2019).

Kott, Sandrine / Droit, Emmanuel (dir.) (2006), Die ostdeutsche Gesellschaft. Eine transnationale Perspektive, Berlin, Christoph Links Verlag.
Lindenberger, Thomas (dir.) (1999), Herrschaft und Eigen-Sinn in der Diktatur. Studien zur Gesellschaftsgeschichte der DDR, Köln/ Weimar/Wien, Böhlau.

Mau, Steffen (2019): Lütten Klein. Leben in der ostdeutschen Transformationsgesellschaft, Berlin, Suhrkamp.

Mesnard, Philippe (2018), " Deux façons de raconter l'histoire ", in Mémoires en jeu, 7, 10-12. (https://www.memoires-enjeu.com/actu/deux-facons-de-raconter-lhistoire/, consulté le 03/11/2019).

Moine, Caroline (2006), « Spur der Zeiten : images documentaires, images de fiction de la DEFA. Un autre regard sur la RDA ? ", in Fabre-Renault, Catherine / Goudin, Élisa / Hähnel-Mesnard, Carola (dir.), La RDA au passé présent. Relectures critiques et réflexions pédagogiques, Paris, Presses Sorbonne Nouvelle, 171-189.

Robin, Régine (2001), Berlin, chantiers, Paris, Stock.

Sabrow, Martin / Eckert Rainer et al. (2007), Wohin treibt die DDRErinnerung? Dokumentation einer Debatte, Göttingen, Vandenhoeck \& Ruprecht.

Steinle, Matthias (2018), «Interroger les images de la RDA. Entretien avec le documentariste Thomas Heise », in Camarade / Guilhamon et al., op. cit., 303-318. 